\title{
Substance Abuse and Suicidal Behavior in Women Ages 30 to 39 Years
}

\author{
Nancy J. Osgood \\ Ameda A. Manetta
}

\begin{abstract}
A growing number of young women abuse substances and engage in suicidal behavior. Combinations of these problems are under-researched. Research that exists includes women of all ages in the samples. This study adds information to the current state of knowledge on these problem behaviors by limiting the sample to women ages 30 to 39 years. A group of 113 women, part of a larger study of 364 women who had received inpatient psychiatric treatment, wasincluded in thisstudy. Findings reveal ed that most of thewomen had madeat least onesuicideattempt and nearly onequarter were in the hospital for a current attempt. Almost $60 \%$ had engaged in substance abuse at some point in their lives. The combination of continued substance abuse and suicidal behavior could result in an early death for some women. Implications for treatment and future research are discussed.
\end{abstract}

Keywords: Women, substance abuse, suicidal behavior

T his study examined the prevalence of substance abuse and suicidal behavior among women in late young adulthood. To date, this population of women has received limited attention (van Wormer, 1999). According to the 1994 report on the treatment of women who abuse alcohol and other drugs, little is known about women who abuse substances because resources for substance abuse research and treatment focus more on men (Center for Substance Abuse Treatment, 1994; Uziel-Miller, Lyons, Kissiel \& Love, 1998). There is a need to expand the focus of substance abuse to include women due to the increased acceptance of alcohol and other drug use and/or abuse in young women, plus a lack of accurate diagnosis and reporting of substance abuse in this group (UzielMiller et al., 1998). Substance abuse has been linked to suicidal behavior in young women. The actual incidence is hard to identify because many attempts go unreported and many women do not seek treatment for an attempt (Crosby, Cheltenham \& Sacks, 1999). Compared to males, females attempt suicide and report suicide ideation more often (Canetto \& Sakinofsky, 1998) and, according to the 1990 National Institute on Alcoholism, female alcoholics complete suicide more frequently than male alcoholics (van Wormer, 1999).

Nancy J. Osgood, Ph.D. is Professor of Gerontology and Sociology, Virginia Commonwealth University/ Medical College of Virginia, Richmond, Virginia. Ameda A. Manetta, Ph.D., M.S.W., L.C.S.W. is Assistant Professor, Winthrop University, Department of Social Work, Rock Hill, SC, 29733. 
Previous studies on substance abuse and suicidal behaviors have included women of all ages (12 years to 80+years) in the samples (Osgood \& Manetta, 1998; Substance Abuse and Mental Health Services Administration, 1999). Some researchers believe that women undergo changes as they age, and there is a difference in life experiences in the 20s, 30s, 40s, and beyond (Bee, 2000). The current study adds to this knowledge base by providing information on women in a distinct age group-30 to 39 years.

\section{ALCOHOL, DRUG ABUSE, PSYCHIATRIC ILLNESS, AND SUICIDE}

Substance abuse encompasses both alcohol and other drug abuse. An estimated 12 million women consume alcohol (Center for Substance Abuse Treatment, 1994). Reporting on the 1992 National Household Survey on Drug Abuse and the 1992 National Household Survey, the Center for Substance Abuse Treatment (1994) stated that $14.5 \%$ of women under age 35 consumed alcohol once a week compared with $10.9 \%$ of women over age 35 . Alcohol abuse occurs more frequently than other drug abuse (Rogers, 1997). The 1992 National Household Survey on Drug Abuse found that 4.4 million women had used illegal drugs and 1.3 million women had used psychotherapeutic drugs for non-medical reasons in the one-month period prior to the study (Substance Abuse and Mental Health Services Administration, 1993). An astounding estimated $80 \%$ of young adults have tried at least one illegal drug (Rogers, 1997). Illicit drug use is highest in the late teens, peaks in the mid-20s, and declines thereafter (Kandel \& Raveis, 1997).

Alcohol and drug abuse are factors in psychiatric illness. Some women who seek substance abuse and mental health services often receive dual diagnosis. Approximately three out of 10 , or $30 \%$ of women with a psychiatric illness, have been treated at some point in their lives for a substance abuse problem (Center for Substance Abuse Treatment, 1994). Depression is often masked by substance abuse (Jarvis \& Copeland, 1997). An estimated seven million women in the United States suffer from clinical depression (Norman \& Lowery, 1995). Studies have shown that the frequencies of women with dual diagnoses of substance abuse and psychiatric problems range between 56\% and 92\% (Haver, 1997).

A link between substance abuse and suicidal behavior has been identified by other researchers (Brabant, Forsyth \& LeBlanc, 1997; Haver, 1997; Jarvis \& Copeland, 1997). In their study of 298 alcoholic patients, Roy, Lamparski, Dejong, Moore \& Linnoila (1990) reported that $30 \%$ of the 85 alcoholic women studied had attempted suicide. There was no difference in the ages (young vs. old) of the women who attempted suicide. In addition, women who attempted suicide reported a greater daily average intake of alcohol than did non-attempters. Well over half (62\%) of these women also met the criteria for a diagnosis of major depression.

Abusing substances is a way of numbing psychic pain; suicide is a way to relieve the pain forever. Many young women engage in both practices. Using alcohol before suicide or a suicide attempt is a common practice. According to the Harvard Mental Health Newsletter (1996), alcohol is involved in almost half of completed suicides. Nielsen, Stenager and Brake (1993) reported 44\% of the women in their study had consumed alcohol either before or during a suicide 
attempt. Women who attempt suicide are likely to make subsequent attempts and are at high risk for eventually killing themselves (Suokas \& Lonnqvist, 1991).

Following the premise that substance abuse declines as women enter the latter part of young adulthood (Kandel \& Raveis, 1997; Substance Abuse and Mental Health Services Administration, 1999) and suicide gradually increases across the lifespan (Canetto \& Sakinofsky, 1998), we focused on women between the ages of 30 and 39 years. In this study, we report on chart review of 113 women discharged after inpatient psychiatric treatment. We followed a descriptive cross-sectional survey design by providing demographic information and information on the prevalence of alcohol and other drug abuse and suicidal behavior. The research questions addressed were:

(1) Is there a positive association between alcohol abuse and suicidal attempts in women 30 to 39 years of age who were treated on an inpatient basis for psychiatric problems?

(2) Is there a positive association between other drug abuse and suicidal attempts in women aged 30 to 39 years of age treated on an inpatient basis for psychiatric problems?

\section{STUDY METHODOLOGY}

Data for this study were gathered at one medical hospital and two state psychiatric facilities in Central Virginia. Criteria for inclusion in the study were (I) discharged patient, (2) female, (3) 30 years of age or older, and (4) admitting diagnosis of one of the depressive disorders, substance use disorder, or substance abuse disorder. Hospital staff used a computer to randomly generate 364 charts from their enumerated charts that met the above criteria during the period October 1994 to October 1996. The total sample included women 30 to 85 years of age (see Manetta, 1997 for a complete description of the sample). The findings reported here are based on a sub-sample of the 113 women who were aged 30 to 39 years.

The methodology consisted of a chart review by a trained research team. The team recorded information from patient charts on a standardized coding instrument. The team was instructed to record information only when it was specifically listed in the patient chart. Inter-rater reliability was $100 \%$. This high rate of inter-rater reliability was attributed to the amount of training the team received (six hours to review 10 charts) and the specificity of the information to be recorded. Demographic information, information on past and current alcohol or other drug abuse, age at first use, and information on past and current suicidal behaviors was generated for each subject.

Current alcohol abuse was defined as being present when the attending physician recorded a primary or secondary DSM-IV diagnosis of alcohol abuse in the chart. Past alcohol abuse was determined by specific notes on the chart referring to a past diagnosis of alcohol abuse, alcohol abuse disorder, or specific notations in the chart of past "alcohol problems." The variable, alcohol abuse, included any past or current alcohol abuse recorded in the chart.

Other drug abuse refers to any psychoactive drug (e.g., over the counter drugs, street drugs, and prescribed drugs). Current other drug abuse was determined to 
be present when the attending physician recorded a primary or secondary DSMIV diagnosis of substance use disorder or substance abuse disorder in the chart. Other past drug abuse was also determined by recording specific chart notations that listed other drug abuse. Other drug abuse was defined as any current or past DSM-IV diagnosis of substance disorder/abuse.

Current suicide and past suicide attempts were defined as any act or repetitive acts deliberately directed toward the self that resulted in physical harm or tissue damage and/or which were attempts to bring about a premature end of life. Suicide attempt(s) were recorded when the behavior was specifically identified in the patient's charts. The only missing data was for marital status. The available marital status data, however, was used to provide demographic information only and not for any of the other analysis.

The demographic data for the 113 women who had alcohol and/ or other drug abuse and/or suicide attempt(s) showed the mean age of the sample was 34.6 years, with a range from 30 to 39 years. The women had an average of 12 years of education. The majority of women were separated or divorced (Table 1). A little more than half of the sample was Caucasian-American and slightly less than half were African-American. The majority of respondents (79.6\%) did not list their religious preference. The preferences that respondents noted were Baptist (9.7\%), Catholic (3.5\%), and other (7.1\%). Due to the sizable degree of missing information, religious preference was not analyzed as a variable.

\begin{tabular}{|lcc|}
\hline \multicolumn{2}{|l|}{ Table 1: Demographic Information on Women Aged 30 to 39 Years (N=113) } \\
\hline Characteristic & $\mathbf{n}$ & $\%$ \\
\hline Marital status & \\
Married & 24 & 22.0 \\
Separated/divorced & 49 & 45.0 \\
Widowed & 2 & 1.8 \\
Single & 34 & 31.2 \\
Ethnicity & & \\
Caucasian-American & 61 & 54.0 \\
African-American & 52 & 46.0 \\
\hline NOTE: ${ }^{\text {ccontained four cases with missing data. }}$ \\
\hline
\end{tabular}

\section{FINDINGS}

To describe the prevalence of substance abuse and suicidal behavior we conducted frequency tests using the Statistical Program for the Social Sciences (SPSS).

Of the 113 women, $30.1 \%$ ( $n=34)$ currently abused alcohol and $44.2 \%(n=50)$ currently abused other drugs (Table 2). More than half $(59.3 \%, n=67)$ abused alcohol and/or other drugs at some point in their lives. The average age that women reported first consuming alcohol was 18 years. Other drug abuse began slightly later, and the average age for first using other drugs was 20 years. The type of drug abused was listed for 31 women and the drug of choice these women identified 
was cocaine $(68 \%, n=21)$. Thirteen percent $(n=4)$ identified abusing prescription drugs.

Suicide ideation was present in $56.6 \%(n=64)$ of the women at the time of their hospitalization. The ideation information was collapsed into one variable to show those who had never had any suicide ideation, those who experienced ideation at one point in time (either in the past or currently), and those who experienced ideation at two points in time (past and currently). Almost half of the women had experienced suicidal ideation at two points in time $(44.2 \%, n=50)$.

Less than half of the women had attempted suicide at some point in their lives $(46.9 \%, n=53)$ and $23.0 \%(n=26)$ were in the hospital for a current suicide attempt.

\begin{tabular}{|lcccc|}
\hline \multicolumn{6}{|c|}{ Table 2: Suicidal Behavior and Substance Abuse of Women Aged 30 to 39 Years } \\
\hline & \multicolumn{3}{c}{ Yes } & \multicolumn{3}{c|}{ No } \\
& N & \% & n & $\%$ \\
\hline Suicide Issues & & & & \\
$\quad$ In Hospital for Current Attempt & 26 & 23.0 & 87 & 77.0 \\
Past Attempts & 53 & 46.9 & 60 & 53.1 \\
Current Ideation & 64 & 56.6 & 49 & 43.4 \\
Past Ideation & 65 & 57.5 & 48 & 42.5 \\
Combined Attempts and Ideation & 79 & 69.9 & 34 & 30.1 \\
Substance Abuse & & & & \\
Current Alcohol Abuse & 34 & 30.1 & 79 & 69.9 \\
Past Alcohol Abuse & 45 & 39.8 & 68 & 60.2 \\
Current Drug Abuse & 50 & 44.2 & 63 & 55.8 \\
Past Other Drugs & 50 & 44.2 & 63 & 55.8 \\
Combined Alcohol and Drug Abuse & 67 & 59.3 & 46 & 40.7 \\
\hline
\end{tabular}

To answer our first question, "Is there a positive association between alcohol abuseand suicidal attempts in women 30 to 39 years of age who were treated on an inpatient basis for psychiatric problems?" we used a chi-square test of independence. The al pha level for the chi-square was set at .10. Table 3 shows a statistically significant association between current al cohol abuse and whether there was a current suicideattempt $\left(X^{2}(1)=3.5, p \leq .05\right)$. However, contrary to our research question, the statistically significant association was in the opposite direction. More women who did not attempt suicide abused alcohol (34.5\%) than those who attempted suicide (15.4\%).

To answer our second question, "Is there a positive association between other drug abuse and suicidal attempts in women aged 30 to 39 years of age who were treated on an inpatient basis for psychiatric problems?" we used a chi-square test of independence. The alpha level for the chi-square was set at .10. We found that there was a statistically significant association for current other drug abuse and whether there was a current suicide attempt $\left(X^{2}(1)=2.5, p \leq .08\right)$. The statistically significant association was in the opposite direction than we had suggested in our research question. The association showed there was not a positive association between current other drug abuse and current suicidal attempts. Abuse of other drugs was more frequent among women who did not attempt suicide (48.3\%) than it was among women who did attempt suicide (30.8\%) (Table 4). 


\begin{tabular}{|llll|}
\hline Table 3: & \multicolumn{4}{l|}{$\begin{array}{l}\text { Current Al cohol Abuse and Current Suicide Attempt for Women Ages } 30 \text { to } 39 \\
\text { Years }\end{array}$} \\
\hline \multicolumn{5}{c|}{ Current Suicide Attempt } \\
No & Yes \\
Current Alcohol Abuse & $\mathbf{( 8 7 )}$ & $\mathbf{( 2 6 )}$ & $\mathbf{X}^{2}$ \\
\hline No $(n=79)$ & $57(65.5 \%)$ & $22(84.6 \%)$ \\
Yes $(n=34)$ & $30(34.5 \%)$ & $4(15.4 \%)$ & $3.5^{*}$ \\
\hline${ }^{*}<05$ & & \\
\hline
\end{tabular}

\begin{tabular}{|llll|}
\hline Table 4: & \multicolumn{4}{l|}{$\begin{array}{c}\text { Current Other Drug Abuse and Current Suicide Attempt for Women Ages } 30 \text { to } \\
\text { 39Years }\end{array}$} \\
\hline \multicolumn{5}{c|}{ Current Suicide Attempt } \\
No & Yes \\
Current Alcohol Abuse & $\mathbf{( 8 7 )}$ & $\mathbf{( 2 6 )}$ & $\mathbf{X}^{2}$ \\
\hline No $(n=63)$ & $45(51.7 \%)$ & $18(69.2 \%)$ \\
Yes $(n=50)$ & $42(48.3 \%)$ & $8(30.8 \%)$ & $2.5^{*}$ \\
\hline${ }^{*} p<08$ & & \\
\hline
\end{tabular}

\section{DISCUSSION}

Many factors contribute to suicidal behavior in youngwomen. We have examined the frequency of substance abuse and suicidal behavior only. While the majority of the women who engaged in alcohol and other drug abuse were not currently in the hospital for a suicide attempt, many of them had attempted suicide in the past. Though the current risk of substance abuse and suicidal behavior may be small, the lifetime risk is much larger, and some of these women are a vulnerable population based on their continued abuse of substances and their combined past and current suicidal behaviors.

Unfortunately, it is not abnormal to find higher rates of substance abuse among women in this age group. Their exposure to risk could be explained by the cohort era. The women were born at a time of changing social values that contributed to the increase in substance abuse (Harrison, 1989). At the time when the average subject in the current study began using illegal drugs (roughly 1985), almost 40\% of young Americans had used some type of illicit drug (Mathias, 1996). Although the frequency of illicit drug use was high in the 1980s, reports also indicate that most illicit drug abuse was declining at that time (Rogers, 1997). It appears that some of the women studied were part of that decline. Nearly $50 \%$ of the women in our sample began using illicit drugs before age 23 , and the rate declined to $39 \%$ of those who continued to abuse drugs at the time of this study. These women had engaged in illicit drug use for 12 to 21 years. We were not able to determine why substance use continued because our sample was selected by a chart review of discharged psychiatric patients and lacked information that may have been available had it been selected from a substance abuse population.

Substances are often used to mask depression. Fortunately, patients with cooccurring psychiatric and substance abuse problems are likely to have their sub- 
stance abuse problems treated (Depression Guideline Panel, 1993) in order to make an accurate psychiatric diagnosis (Weiss, Griffin \& Mirin, 1992). The psychiatric diagnosis most often associated with suicidal behavior is depression (Cohen, Lavell, Rich \& Bromet, 1994). Treating substance abuse, then making a psychiatric diagnosis would be helpful to the women in our study. Once their alcohol abuse is under control, their psychiatric problems can be addressed. However, as pointed out previously, women often abuse prescription medications and the alcohol abuse could be replaced by abuse of psychotropic medications. We can speculate that this may have occurred because, in our study, we found that over time, alcohol abuse had declined but other drug abuse had remained the same. Thus, we did not support the findings that alcohol abuse is more prevalent than other drug abuse that other researchers have reported (Rogers, 1997). The length of time substances were abused, however, was consistent with other studies that report current substance abuse in dually diagnosed patients of long duration (Dixon, McNary \& Lehman, 1998). Others have reported that alcohol abuse/ use is present in some women who engage in suicidal behaviors. Our findings were congruent with theirs (Haver, 1997; Jarvis \& Copeland, 1997; Osgood \& Manetta, 1998). In this study, we found that young women who abused alcohol and/or other drugs were less likely to attempt suicide compared to young women who did not abuse alcohol and/or other drugs. Based on the limited research available on this population, we were surprised at these findings. It is possible for young women to use alcohol and/ or other drugs to self-medicate for depression and, therefore, they are less likely to engage in suicidal behaviors.

This was not a longitudinal study. It is possible that self-medicating with alcohol and/or other drugs is one way to combat depression in the younger years; however, as these women grown older, the alcohol and other drugs could actually become a contributing factor in later suicidal behavior. When we looked at older women from this sample, we found a positive relationship between abusing alcohol and suicidal behavior and abusing other drugs and suicidal behavior (Osgood $\&$ Manetta, 1998). Another possible explanation for the current findings is that some young women, in spite of their alcohol and drug abuse, do not turn to suicide because they have other internal or external factors that allow them to be resilient.

\section{Treatment Implications}

Treatment modalities for combined substance abuse problems and psychiatric problems, including suicidal behaviors, are scarce (Cornelius, Salloum, Lynch, Clark \& Mann, 2001). Therefore, treatment should be based on the combined known indicators of each diagnosis. It is known that the intent to kill oneself rises with each suicide attempt and depression is often present before and after the attempt (Hamdi, Amin \& Mattar, 1991). In addition, many women claim depression is a trigger for their drug abuse (Griffin, Weiss \& Mirin, 1989). If a woman seeks treatment for depression and suicidal behavior and her substance abuse is not revealed, she may be given psychotherapeutic medications to treat the depression. When combined with alcohol or other illicit drugs, these medications can create a lethal cocktail. Also, many psychotherapeutic drugs are addictive 
and, if the underlying causes are not treated, a cycle of drug abuse and suicidal behavior often occurs. It is essential that clinicians working with young women provide concurrent treatment for both substance abuse problems and psychiatric problems. Furthermore, a longer length of time is needed to treat dually diagnosed women than the short time used to treat women diagnosed with substance abuse only (Center for Substance Abuse Treatment, 1994). Thus, the current cost saving measures of brief therapy will not work as well with this population.

\section{CONCLUSION}

In this study, we have provided additional information to bolster the scant knowledge that exists on dually diagnosed women. Substance abuse is present in many suicidal attempts and completed suicides. People who eventually complete suicide have often made a previous suicide attempt. For women, the chance of completing suicide increases with age. In this research, we have identified the prevalence of substance abuse in women in the late part of young adulthood; and we have identified the fact that more than half of these women have engaged in suicidal behavior at some time in their lives. We have suggested treatment implications for professionals who provide services to the women.

This study has some limitations. First, data were gathered by chart review. Chart review is an acceptable data source and is often used when availability to subjects is limited (Carmen, Rieker \& Mills, 1984). Second, information may have been missing from the charts. Nevertheless, the information in the charts was originally compiled for treatment purposes, thus, the researchers relied on the professionals at the psychiatric facilities to obtain a comprehensive history of the problems being treated. Third, most of the information was self-reported by patients and selective information may have been given. Providing selective information that portrays peopleas having desirable social qualities is a limitation in many survey research studies. The women in this study had been in a psychiatric facility; consequently, they were already outside of the norms of social behavior. Therefore, we believed the threat to internal validity of obtaining selective information was low. Finally, cross-sectional research focuses on what exists at one point in time and, thereby, limits the generalizability of the results. The reader needs to be aware that this research is only generalizable to the population studied. Nonetheless, only limited information on substance abuse and suicidal behavior in young women is currently available; and the available information tends to include both genders in the results. Thus, the exclusive focus on young women in this study adds valuable information to the knowledge base of professional helpers.

There is a need for future studies. A longitudinal study on women with suicidal behavior and substance abuse could describe the behaviors that occur as these women reach different ages. Other studies are needed to identify factors that underlie substance abuse and suicidal behaviors among women of various ages. Women undergo diverse life events, have dissimilar perspectives toward life, and have different coping skills at different ages. It is only by teasing out the differences in the factors that contribute to suicidal behavior and/ or substance abuse that we can begin to understand and build the knowledge base necessary to 
describe incremental age differences between substance abuse and suicidal behaviors of women. Therefore, future examinations of this age group could focus on particular resiliency factors that protect this vulnerable population of women from suicidal behavior.

\section{References}

Bee, H.L. (2000). Thejourney of adulthood ( $4^{\text {th }}$ ed.). Upper Saddle River, NJ: Prentice Hall.

Brabant, S., Forsyth, C.J., \& LeBlanc, J.B. (1997). Childhood sexual trauma and substance misuse: A pilot study. SubstanceUseand Misuse, 32(10), 1417-1431.

Canetto, S.S., \& Sakinofsky, I. (1998). The gender paradox in suicide. Suicideand Life-Threatening Behavior, 28(1), 1-23.

Carmen, E., Rieker, P.P., \& Mills, T. (1984). Victims of violence and psychiatric illness. American Journal of Psychiatry, 141, 373-383.

Center for Substance Abuse Treatment. (1994). Practical approaches in thetreatment of women who abuse alcohol and other drugs. Rockville, MD: Department of Health and Human Services, Public Health Service.

Cohen, S., Lavell, J., Rich, C.L., \& Bromet, E. (1994). Rates and correlates of suicide attempts in first-admission psychotic patients. Acta Psychiatrica Scandinavica, 90, 167-171.

Cornelius, J.R., Salloum, I.M., Lynch, K., Clark, D.B., \& Mann, J.J. (2001). Treating the substance-abusing suicidal patient. Annals of the New York Academy of Sciences, 932, 78-90.

Crosby, A.E., Cheltenham, M.P., \& Sacks, J.J. (1999). Incidence of suicidal ideation and behavior in the United States, 1994. Suicideand Life-Threatening Behavior, 29(2), 131-140.

Depression Guideline Panel. (1993). Depression in primary care: Detection, diagnosis and treatment. Quick Reference Guidefor Clinicians, Number 5, Rockville, MD: U.S. Department of Health and Human Services, Public Health Service, Agency for Health Care Policy and Research. AHCPR Publication No. 930052.

Dixon, L., McNary, S., \& Lehman, A.F. (1998). Remission of substance use disorder among psychiatric inpatients with mental illness. American Journal of Psychiatry, 155(2), 239-243.

Griffin, M.L., Weiss, R.D., \& Mirin, S.M. (1989). A comparison of male and female cocaineabusers. Archives of General Psychiatry, 46, 122-126.

Hamdi, E., Amin, Y., \& Mattar, T. (1991). Clinical correlated of intent in attempted suicide. Acta Psychiatrica Scandinavica, 83(5), 406-411.

Harrison, P.A. (1989). Women in treatment: Changing over time. The International Journal of the Addictions, 24(7), 655-673.

Harvard Mental Health Newsletter. (1996, November). Suicide. (part 1), 13(5), 1,5.

Haver, B. (1997). Screening for psychiatric co-morbidity among female alcoholics: The use of a questionnaire (SCL-90) among women early in their treatment program. Alcohol \& Alcoholism, 32(6), 725-730.

Jarvis, T.J., \& Copeland, J. (1997). Child sexual abuse as a predictor of psychiatric co-morbidity and its implications for drug and alcohol treatment. Drug and Alcohol Dependence, 49, 61-69.

Kandel, D.B., \& Raveis, V.H. (1997). Teens who use drugs to "fit in" more likely to stop for same reason. A collection of NIDA Notes: Articles that Address Women, Gender Differences, and Drug Abuse, June 1997. Rockville, MD: National Institute on Drug Abuse, National Institutes of Health, NN0013.

Manetta, A.A. (1997). Factors in suicidal ideation and attempts in women aged 40 to 64 years who have been admitted to in-patient psychiatric facilities. (Doctoral dissertation, Virginia Commonwealth University, 1997). Dissertation Abstracts International, 58, 034.

Mathias, R. (1996, January/ February). Students' use of marijuana, other illicit drugs, and cigarettes continued to rise in 1995. A Collection of NIDA Notes, 11(1). Retrieved from: http:// www.drugabuse.gov/NIDA_Notes/NNVol1N1/StudentSurvey.html 
Nielsen, A.S., Stenager, E., \& Brake, U.B. (1993). Attempted suicide, suicidal intent, and alcohol. Crisis, 14(1), 32-38.

Norman, J., \& Lowery, C.E. (1995). Evaluating inpatient treatment for women with clinical depression. Research on Social Work Practice, 5(1), 10-19.

Osgood, N.J., \& Manetta, A.A. (1998). Alcohol abuse, drug abuse, and suicidal behavior in older women. The Southwest Journal on Aging, 14(1), 85-90.

Rogers, S. (1997). NIDA's high school senior survey also provides data on college students' drug use. A Collection of NIDA Notes: Articles that Address Women, Gender Differences, and Drug Abuse, June 1997. Rockville, MD: National Institute on Drug Abuse, National Institutes of Health, NN0013.

Roy, A., Lamparski, D., Dejong, J., Moore, V., \& Linnoila, M. (1990). Characteristics of alcoholics who attempt suicide. American Journal of Psychiatry, 147(6), 761-765.

Suokas, J., \& Lonnqvist, J. (1991). Outcome of attempted suicide and psychiatric consultation: Risk factors and suicide mortality during a five-year follow-up. Acta Psychiatrica Scandavia, 84, 545-549.

Substance Abuse and Mental Health Services Administration. (1993). National Household Survey on Drug Abuse: Population Estimates. 1992. Rockville, MD, 55.

Substance Abuse and Mental Health Services Administration. (1999). Summary findings from the 1998 national household survey on drug abuse. Rockville, MD. DHHS Publication No. (SMA) 99-3328.

Uziel-Miller, N.D., Lyons, J.S., Kissiel, C., \& Love, B.S. (1998). Treatment needs and initial outcomes of a residential recovery program for African-American women and their children. The American Journal on Addictions, 7(1), 43-50).

van Wormer, K. (1999). Al coholism treatment: A social work perspective Chicago, IL: Nelson-Hall.

Weiss, R.D., Griffin, M.L., \& Mirin, S.M. (1992). Drug abuse as self-medication for depression: An empirical study. Journal of Drug and Alcohol Abuse, 18(2), 121-129.

\section{Author's Note:}

Address correspondence to: Ameda A. Manetta, Ph.D., M.S.W., L.C.S.W., Assistant Professor, 128 Bancroft Building, Dept. of Social Work, Winthrop University, Rock Hill, SC 29733, USA. E-mail: manettaa@winthrop.edu. 